\title{
nouveau dispositif expérimental pour l'étude de la rhéologie des roches
}

\author{
par \\ J. Fine, S.M. Tijani, G. Vouille \\ Centre de Mécanique des Roches Fontainebleau
}

\section{Introduction}

Indispensable pour le dimensionnement et létude de la stabilité des ouvrages conçus en massifs rocheux, une connaissance précise des lois de comportement des roches nécessite des moyens d'investigation de plus en plus perfectionnés qui permettent l'étude du comportement élastoplastique (courbe intrinsèque) par des \& essais triaxiaux " ainsi que l'étude de l'influence du facteur temps (viscoélasticité et élastoviscoplasticité) par des essais d'écrouissage, de fluage et de relaxation.

L'essai triaxial, très utilisé pour mesurer la limite d'élasticité présente deux inconvénients non négligeables.

Premièrement, pour obtenir la courbe intrinsèque d'une roche (enveloppe des cercles de Mohr) on doit utiliser de nombreux échantillons (détruits à la fin de l'essai) ; deuxièmement, la détermination d'une limite d'élasticité (contrainte) par un « essai triaxial » nécessite la mesure d'allongements (déformations), pour laquelle on utilise le plus souvent des jauges électriques qui, sous pression, sont peu fiables (dérive importante en fonction du temps) et, avec certaines roches, peuvent se décoller en cours d'essai.

Le Dispositif de Relaxation Biaxiale et Isotherme (DRBI), objet du présent exposé, permet d'obtenir les mêmes résultats qu'une série d'essais triaxiaux et ce, sans présenter les deux inconvénients ci-dessus, en fournissant des renseignements supplémentaires sur la rhéologie des roches.

\section{Principe de fonctionnement du dispositif}

\subsection{Chargement biaxial}

Comme lors d'un essai triaxial, un échantillon cylindrique est placé dans une cellule d'acier où il est soumis à une pression axiale (notation: Q) et à une pression latérale (notation: P). Pour un tel chargement, l'éprouvette est soumise à un champ de contraintes uniforme dans l'espace et isotrope dans toute section droite (les 2 contraintes principales latérales sont égales).

Cependant, contrairement à l'essai triaxial pour lequel usuellement la pression axiale est plus forte que la pression latérale (régime de compression), avec le DRBI, les essais sont réalisés aussi bien en régime de traction qu'en régime de compression.

\subsection{Régulation thermique}

La cellule contenant l'éprouvette est placée dans une enceinte calorifugée afin d'éviter les perturbations des mesures causées par les fluctuations de la température ambiante et d'étudier l'influence du paramètre température sur les caractéristiques mécaniques des roches.

\subsection{Fonctionnement}

L'une des deux pressions ( $P, Q$ ) est pilotée manuellement ou automatiquement selon un historique préprogrammé, l'autre est asservie de sorte que la longueur de l'échantillon reste constante durant l'essai (déformation axiale constante : « relaxation »).

Compte tenu de la condition géométrique d'invariance de la longueur de l'éprouvette, l'historique de la pression asservie est fonction de l'historique de la pression pilotée et ce suivant une loi qui dépend de la nature du matériau constituant l'échantillon. Ainsi l'analyse de la relation entre les historiques des deux pressions permet de renseigner sur les lois physiques qui régissent le comportement d'une roche.

\section{Analyse des essais}

\subsection{Chargement quasistatique : (fig, 1)}

On suppose que l'on pilote la pression latérale $(P)$ en la faisant croitre à vitesse suffisamment faible pour que l'échantillon soit à tout instant dans un état d'équilibre caractérisé par la pression latérale (P) et la pression axiale (Q).

Si l'on représente cet état d'équilibre par un point d'abscisse $P$ et d'ordonnée $Q$ (diagramme $(P, Q)$ ) on s'apercoit que ce point décrit une certaine courbe $(Q$, pression 
asservie, est fonction de P) dont la forme dépend de la nature de la roche.

Roche elastique : cœefficient de Poisson (fig. 1)

La plupart des roches ont un comportement élastique tant que la pression latérale n'a pas atteint un certain seuil (caractéristique mécanique de la roche). Si l'on écrit la relation entre la déformation axiale $(\varepsilon$ : variation relative de la longueur de l'échantillon) et les pressions $\mathrm{P}$ et $\mathrm{Q}$ en désignant par E le module d'Young de la roche et par v son ccefficient de Poisson, il vient :

$\varepsilon=\frac{1}{E}(-\mathrm{Q}+2 v \mathrm{P})$

Or la longueur de l'échantillon est constante durant l'essai. D'où $\varepsilon=0$ et par conséquent $Q=2 v P$

On en déduit qu'au début de l'essai ( $P$ inférieure à un certain seuil), la relation entre $Q$ et $P$ (Linéaire) est représentée dans le diagramme (P,Q) par une portion de droite dont la pente est le double du coefficient de Poisson de la roche: d'oú une méthode pour déterminer celui-ci sans faire appel à des mesures extensométriques.

Roche élastoplastique: courbe intrinsèque (fig. 1 et 2)

Quand la pression latérale dépasse un certain seuil, le point représentatif dans le diagramme (P,Q) quitte la droite quî caractérise la phase élastique pour décrire une portion de courbe $(\Gamma)$ dont chaque point représente un état d'équilibre limite.

Cet état d'équilibre limite est représenté aussi, parfois dans l'espace des contraintes principales (le point représentatif est alors sur une arête de la surface limite d'élasticité, arête de compression si $Q \geqslant P$ et de traction si $P \geqslant Q$ ) et le plus souvent dans le diagramme de Mohr par un cercle (diamètre : $|\mathrm{P}-\mathrm{O}|)$ tangent à la courbe intrinsèque. Ainsi, à chaque point $(P, Q)$ de la courbe $(\Gamma)$ correspond un cercle de Mohr et l'enveloppe de cette famille de cercles n'est autre que la courbe intrinsèque de la roche qui se trouve tracée en n'utilisant qu'un seul échantillon alors que par la méthode des "essais triaxiaux " pour chaque cercle de Mohr il faut un échantillon (détruit à la fin de l'essai). Un calcul simple montre que quand la courbe intrinsèque est une droite $(\Delta)$ (critère de Coulomb-Tresca ou Drucker-Mises), la courbe ( $\Gamma$ ) est une droite et que la connaissance de la valeur à l'origine et de la pente de cette droite $(\Gamma)$ permet de calculer la valeur à l'origine (cohésion) et la pente (tg $\emptyset$ : $\emptyset$ : angle de frottement) de la droite $(\Delta)$.

\subsection{Relaxation (fig. 3 et 4)}

On part d'un état d'équilibre limite $(P, Q)$ (appartenant à la courbe $(\Gamma))$ et on supprime l'asservissement pour permettre à la longueur de l'échantillon de varier.

On peut alors agir sur les pressions $\mathrm{P}$ et $\mathrm{Q}$ indépendamment l'une de l'autre.

Si l'on diminue la pression axiale $(Q)$ en maintenant constante la pression latérale $(P)$ (dans la pratique cette opération est effectuée suffisamment rapidement pour ne pas laisser à l'échantillon le temps de subir des déformations différées), cette baisse de la pression axiale perturbe l'équilibre limite et conduit à un état de contrainte hors du domaine d'élasticité. Par conséquent si on maintient ce chargement, sans rétablir l'asservissement, la longueur de l'échantillon qui a déjà subi une variation instantanée (élastique) se met à augmenter (fluage).

Par contre, si l'on rétablit l'asservissement, pour s'opposer à cette augmentation différée de la longueur de l'échantillon, la pression axiale se mettra à augmenter (relaxation). Si l'on représente dans un diagramme en abscisses le temps et en ordonnées la pression axiale on obtient une courbe dont la forme dépend de la nature de la roche et sur laquelle on peut ajuster (par " moindre carrés ») des paramètres viscoplastiques de la roche.

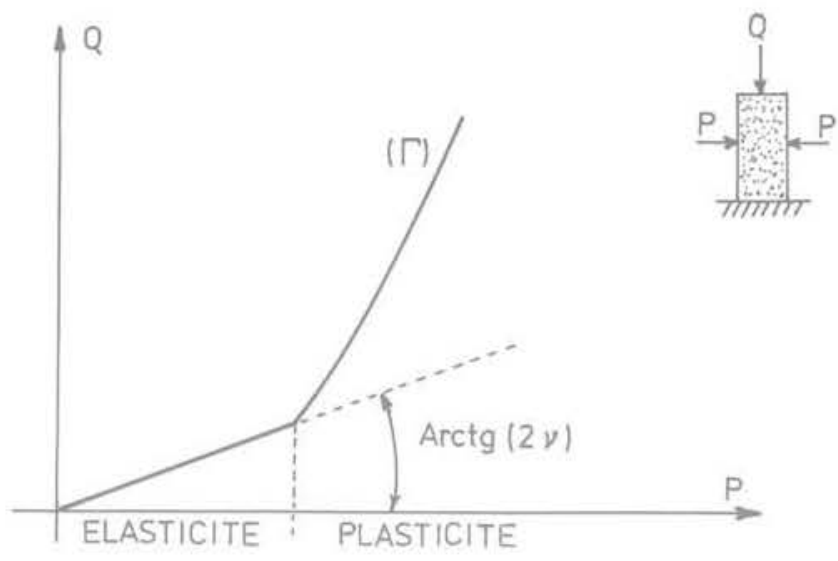

Fig. 1 Diagramme $(P, Q)$

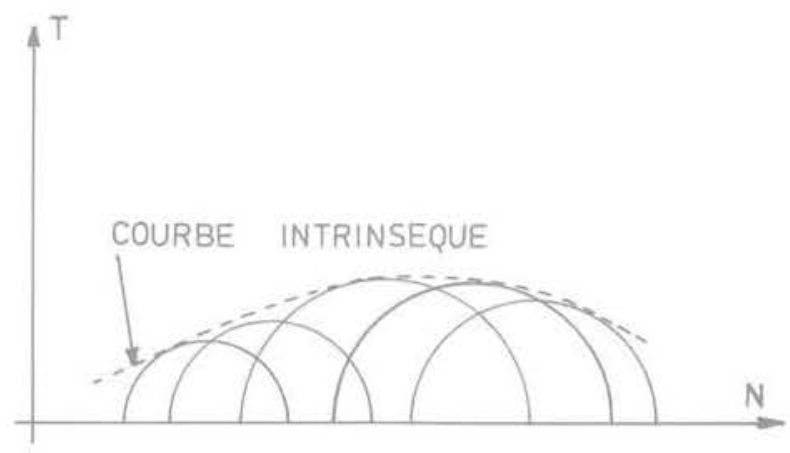

Fig. 2 Diagramme de Mohr

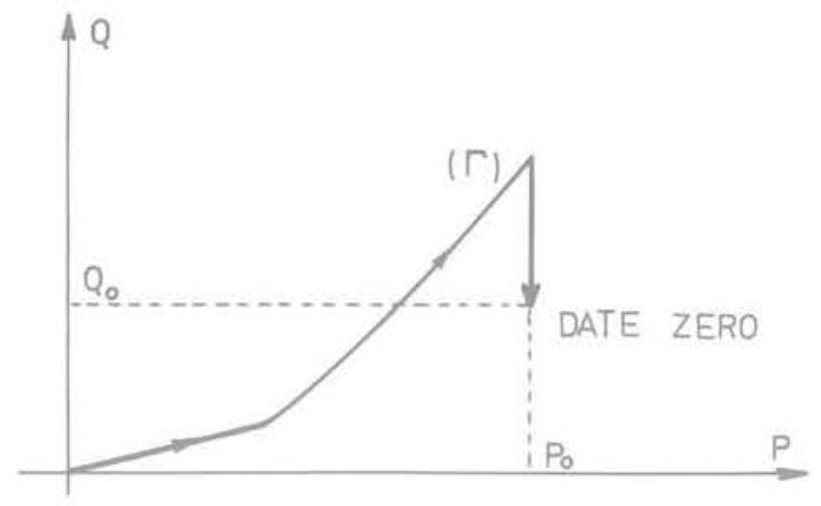

Fig. 3 Mise en charge avant relaxation



Fig. 4 Relaxation. $P=P_{0}$ 


\subsection{Conclusion}

On vient de voir que des historiques simples de la pression pilotée (évolution à vitesse lente ou pression maintenue constante) permettent d'accéder à de nombreuses caractéristiques mécaniques de la roche étudiée. D'autres caractéristiques peuvent être déterminées en donnant à la pression pilotée des historiques plus complexes (évolution à différentes vitesses, cycle de charge-décharge...) qui permettent d'étudier divers phénomènes tels que l'écrouissage et la fatigue de la roche.

\section{Description du dispositif}

Cinq parties principales composent le Dispositif de Relaxation Biaxiale et Isotherme (fig. 5).

\subsection{Cellule de compression biaxiale: (fig. 6)}

\section{Géométrie de la cellule}

De forme cylindrique, la cellule est prévue pour recevoir un échantillon d'environ $65 \mathrm{~mm}$ de diamètre et de $130 \mathrm{~mm}$ de longueur. Fabriquée en acier, elle présente comme toute "cellule triaxiale \& quatre parties principales :

- un corps, possèdant sur sa surface latérale une arrivée d'huile (pression latérałe) et fileté à ses deux extrémités. La jaquette de protection (en silastène), entourant l'échantillon et permettant la transmission de la pression latérale, se trouve à lintérieur du corps

- un chapeau qui vient se visser sur la partie supérieure du corps. II est constitué de deux étages séparés par une membrane en caoutchouc. l'étage supérieur étant muni d'une arrivée d'huile (pression axiale)

- un piston inférieur fixe qui est relié au corps par l'intermédiaire d'une partie filetée et sur lequel repose l'échantillon

- un piston supérieur mobile qui occupe l'espace séparant la membrane et le haut de l'échantillon et qui transmet la pression axiale exercée par l'huile sur la membrane.

\section{Différence avec la cellule classique}

La hauteur du piston mobile (donc celle de l'étage inférieur du chapeau) a été agrandie. Deux boulons vissés au piston traversent (en deux points diamétralement opposés) l'étage inférieur du chapeau avec un certain jeu permettant au piston une course de l'ordre de $5 \mathrm{~mm}$. Le mouvement du piston (grâce aux boulons et à un montage adéquat de pièces secondaires) est transmis à la pointe d'un comparateur dont le corps est rendu solidaire du piston inférieur.

\subsection{Comparateur}

Le comparateur possède deux contacts électriques amovibles qui permettent, toutes les fois que l'aiguille quitte une certaine plage, la fermeture d'un circuit électrique comportant un relais qui commande la pompe délivrant la pression asservie (aussi bien pour la charge : moteur, que pour la décharge: électrovanne).

\subsection{Pompes et accumulateurs}

Deux pompes indépendantes délivrent les pressions axiale et latérale. Pendant que l'une est pilotée manuellement ou automatiquement (programmateur), l'autre est asservie (comparateur). Des accumulateurs à azote sont placés entre les pompes et la cellule pour assurer une régularité dans la mise en charge, nécessaire pour mener à bien les essais.

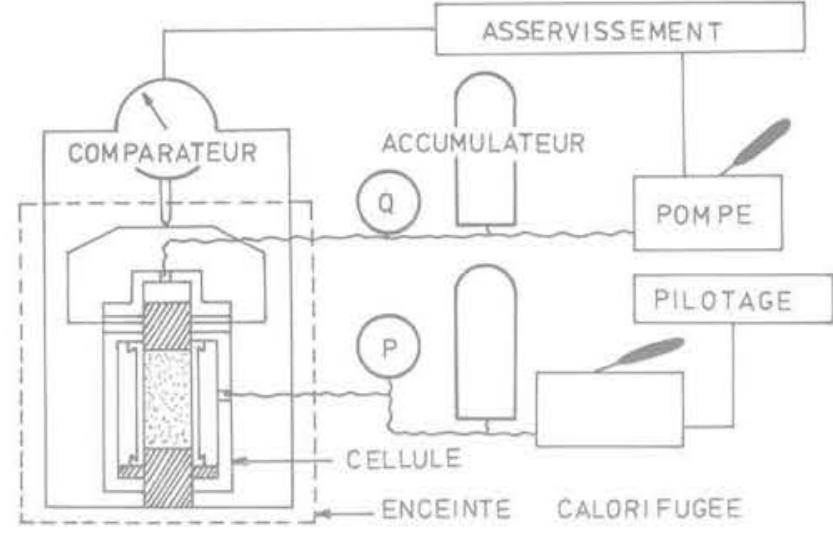

Fig. 5 D.R.B.I. (schéma de principe)

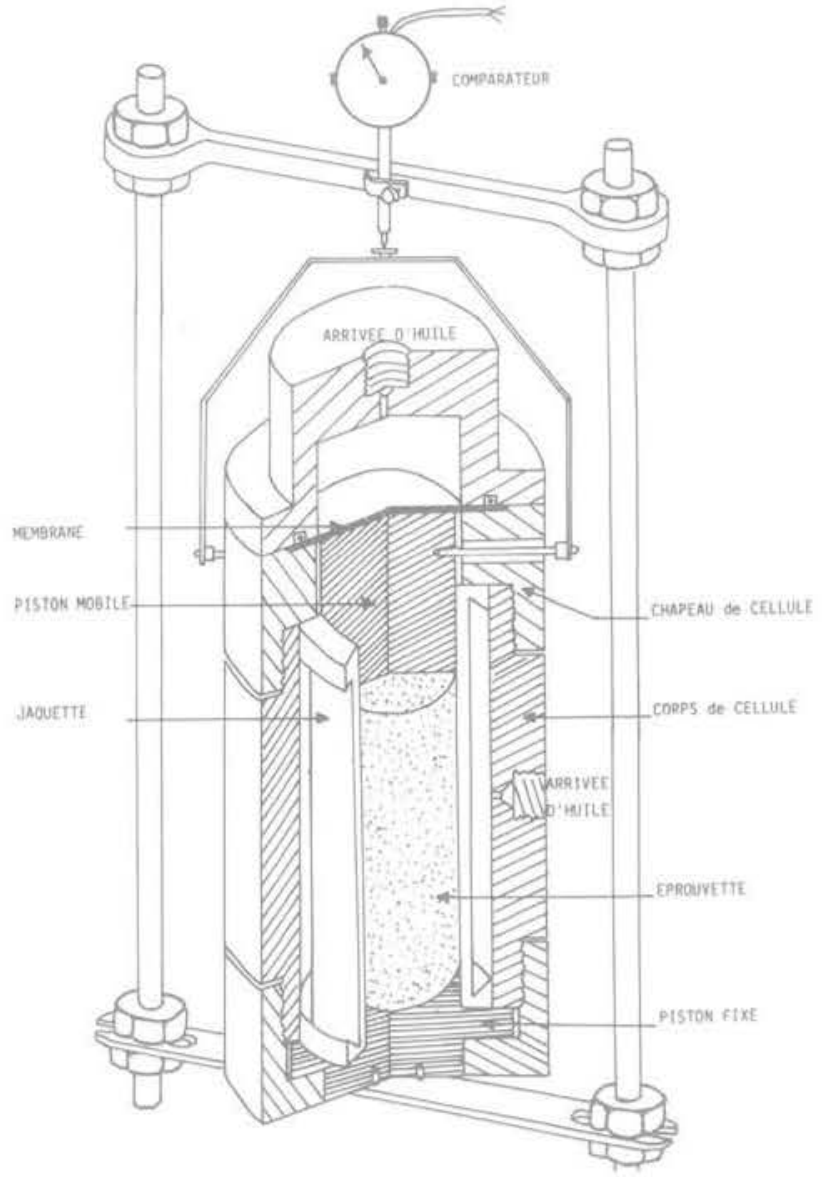

Fig. 6 Cellule de compression biaxiale

\subsection{Programmateur}

Un montage électronique permet de piloter une des deux pompes qui fournit ainsi une pression pilotée qui varie selon un schéma préprogrammé (variation à vitesse constante, cycles de charge-décharge...) 


\subsection{Système de régulation de la température}

La cellule est placée dans une enceinte calorifugée où une température, uniforme dans l'espace et constante dans le temps, est assurée par une huile spéciale, très fluide, véhiculée par une petite pompe et chauffée dans une autre enceinte à l'aide d'une résistance électrique. Deux thermomètres à seuils, placés dans chacune des enceintes, permettent de fermer deux relais qui commandent l'un la résistance électrique et l'autre la pompe.

Outre ces cinq parties, on envisage de munir le DRBI d'un capteur de déplacement pour la mesure de la déformation latérale permettant ainsi l'accès à d'autres caractéristiques mécaniques de la roche, ainsi que de deux capteurs de pressions, l'ensemble de ces capteurs étant relié à une " acquisition de données " permettant d'enregistrer les mesures (déformations latérales, pressions et temps) sur papier et sur bande perforée, ce qui facilitera les traitements automatiques (tracés de courbes, ajustement de paramètres...)

\section{Conclusion}

Bien que d'une structure complexe, le DRBI n'en est pas moins un dispositif souple qui, fondé sur un principe de base simple (condition géométrique d'invariance de la longueur de l'éprouvette durant les essais), permet d'effectuer une série d'essais sur un même échantillon (chargement quasistatique, relaxation, décharge...), essais dont les résultats décrivent en grande partie la loi de comportement d'une roche (cœefficient de Poisson, courbe intrinsèque, paramètres viscoplastiques...).

Le Dispositif de Relaxation Biaxiale Isotherme est utilisé actuellement pour l'étude de la rhéologie du sel gemme, des anhydrides et des argiles.

\section{Références bibliographiques}

FINE J. - TIJANI S.M. - VOUILLE G. - BOUCLY P. - Détermination expérimentale de quelques paramétres êlastoviscoplastiques des Roches. Application aux cavités de stockage du gaz en couches salines profondes. $4^{\circ}$ Congrès International de Mécanique des Roches 1979. MONTREUX (SUISSE)

TIJANI S.M. - Rhéologie du Sel gemme. - (rapport interne du Centre de Mécanique des Roches R 75/15) Influence de la température sur les propriétés mécaniques du sel germme - (rapport interne du Centre de Mécanique des roches $R$ 76/2)

VOUILLE G. - Etude de linfluence de la température sur les caractéristiques élastoviscoplastiques du sel de TERSANNE (rapport interne du Centre de Mécanique des Roches SE 78/7) 